\title{
Modified Analog Transmission Scheme for Distributed Detection
}

\author{
Xiangyang LIUa, Tenglong JIANG and Zhijie MAO \\ Dept. of Information Transmission, Xi'an Communications Institute, Xi'an, \\ Shaanxi Province, China \\ aliuxiangyangdr@gmail.com
}

\begin{abstract}
In this paper, we consider the design of a modified analog transmission scheme for binary distributed detection systems in which the compressed information made by the local sensors are sent to the fusion center (FC) over parallel Rayleigh fading channels. For complexity concerns, we focus on the equal gain combiner fusion rules. Simulation results show that, without increasing extra transmission costs, the proposed transmission scheme can achieve significant performance gain not only over that of the widely adopted binary transmission scheme but also over that of the simple analog transmission scheme.
\end{abstract}

Keywords: distributed detection; analog transmission; wireless sensor network; data fusion

\section{Introduction}

A distributed detection system usually consists of multiple sensors and a fusion center (FC). In wireless sensor networks, the local decisions are sent over wireless channels and are prone to transmission errors. This motivates the needs of distributed detection algorithms that take into account the effects of the channels [1-5]. Fusion of binary decisions transmitted over fading channels has prevailed.

Although digital transmission predominates in modern communication system, analog one has still its own position. Considering that the multi-level or analog compressed information made by the local sensors are more informative than the binary decision, proper analog transmission scheme may improve the detection 
performance of the distributed detection system. In [6], local sensor decisions are transmitted by means of analog relay amplifier processing. For both deterministic and stochastic Gaussian signals, asymptotic performance in a large sensor system is analyzed by deriving the error exponents. $\operatorname{In}[7,8]$, each distributed node performs analog-relay amplifier local processing on its observation and transmits locally processed data to the fusion center over a wireless channel.

By simulations, we found that, the system using binary transmission perform better than that using analog transmission does, which explains why the former is prevailing. Theoretically, however, analog transmission can convey more information than binary one. Gastpar[9] showed that for a symmetric sensor network with no fading, analog transmission achieves the optimal performance theoretically attainable. Therefore, we devised a modified analog transmission scheme with significant performance gain over the binary transmission.

\section{Transmission Scheme}

We assume a frequency-nonselective slow-fading model for each channel, i.e., the channel remains unchanged during the transmission of the information of each sensor.

Binary Transmission. In this case, each local sensor transmits its local decision $u_{k}$ to the FC and the output of the channel for the k-th sensor[2] can be denoted by $r_{k}=\sqrt{P_{r k}^{f c}} h_{k} u_{k}+w_{k}$. The SNR of $r_{k}$ is given by $\lambda_{c k}^{B}=\frac{\mathbb{E}\left[\left(\sqrt{P_{r k}^{f c}} h_{k} u_{k}\right)^{2}\right]}{\mathbb{E}\left[\left(w_{k}\right)^{2}\right]}=\frac{\mathbb{E}\left[h_{k}^{2}\right] P_{r k}^{f c}}{\sigma_{w_{k}}^{2}}=\frac{P_{r k}^{f c}}{\sigma_{w_{k}}^{2}}$. Obviously, local binary quantization will lose some useful information. Therefore, a modified analog transmission is given in the following. 
Analog amplifier transmission. Each local test statistic $S_{k}$ is amplified by $\sqrt{P_{r k}^{f c}}$, the output of the channel for the k-th sensor is given by $r_{k}=\sqrt{P_{r k}^{f c}} h_{k} S_{k}+w_{k}$. The SNR of $r_{k}$ can be denoted by $\lambda_{c k}^{A}=\frac{\mathbb{E}\left[\left(\sqrt{P_{r k}^{f c}} h_{k} s_{k}\right)^{2}\right]}{\mathbb{E}_{[}\left[\left(w_{k}\right)^{2}\right]}=\frac{\mathbb{E}\left[h_{k}^{2}\right] \mathbb{E}\left[s_{k}^{2}\right] P_{r k}^{f c}}{\sigma_{w_{k}}^{2}}=\frac{P_{r k}^{f c}}{\sigma_{w_{k}}^{2}} \frac{\left(1+\lambda_{k}\right)^{2} M(M+1)}{(R-1)(R-2)}$

Modified Analog amplifier transmission. Each local test statistic $S_{k}$ is modified according to $s_{k}^{\prime}=\left\{\begin{array}{cc}s_{k} / \tau_{k}, & s_{k} \geq \tau_{k} ; \\ -1, & s_{k}<\tau_{k} .\end{array}\right.$. Hence, the output of the channel for the k-th sensor is given by $r_{k}=\sqrt{P_{r k}^{f c}} h_{k} s_{k}^{\prime}+w_{k}$, where $w_{k}$ is zero mean Gaussian noise with variance $\sigma_{w_{k}}^{2}$, and $h_{k}$ is the gain of a real valued Rayleigh fading channel with the PDF given by $f\left(h_{k}\right)=2 h_{k} e^{-h_{k}^{2}}, h_{k} \geq 0$. The SNR of $r_{k}$, under $H_{1}$, is given by

$$
\lambda_{c k}^{M A}=\frac{\mathbb{E}\left[\left(\sqrt{P_{r k}^{f c}} h_{k} s_{k}^{\prime}\right)^{2}\right]}{\mathbb{E}\left[\left(w_{k}\right)^{2}\right]}=\frac{P_{r k}^{f c}\left(1+\lambda_{k}\right)^{2} M(M+1)}{\sigma_{w_{k}}^{2} \tau_{k}^{2}(R-1)(R-2)} P_{l d}\left(\tau_{k}, \lambda_{k}, M+2, R-2\right)+\frac{P_{r k}^{f c}}{\sigma_{w_{k}}^{2}}\left[1-P_{l d}\left(\tau_{k}, \lambda_{k}, M, R\right)\right]
$$

The probability density function of $r_{k}$ is

$$
f\left(r_{k} / h_{k}, s_{k}, P_{r k}^{f c}\right)=\frac{1}{\sqrt{2 \pi \sigma_{w_{k}}^{2}}} \exp \left[-\frac{\left(r_{k}-\sqrt{P_{r k}^{f c}} h_{k} s_{k}^{\prime}\right)^{2}}{2 \sigma_{w_{k}}^{2}}\right]
$$




$$
\begin{aligned}
& f\left(r_{k} / H_{1}\right)=\int_{0}^{+\infty} \frac{2 h_{k} e^{-h_{k}^{2}}}{\sqrt{2 \pi \sigma_{w_{k}}^{2}}} \exp \left[-\frac{\left(r_{k}+\sqrt{P_{r k}^{f c}} h_{k}\right)^{2}}{2 \sigma_{w_{k}}^{2}}\right] d h_{k}\left(1-\sum_{n=1}^{M} \frac{\tau_{k}^{n-1} \Gamma(R+n-1)\left(1+\lambda_{k}\right)^{R}}{\Gamma(R)\left(1+\lambda_{k}+\tau_{k}\right)^{R+n-1}}\right) \\
& +\int_{0}^{+\infty} \int_{\tau_{k}}^{+\infty} \frac{2 h_{k} e^{-h_{k}^{2}}}{\sqrt{2 \pi \sigma_{w_{k}}^{2}}} \exp \left[-\frac{\left(r_{k}-\sqrt{P_{r k}^{F c}} h_{k} s_{k} / \tau_{k}\right)^{2}}{2 \sigma_{w_{k}}^{2}}\right] \frac{s_{k}^{M-1}\left(1+\lambda_{k}\right)^{R} \Gamma(M+R)}{\left(1+s_{k}+\lambda_{k}\right)^{M+R} \Gamma(M) \Gamma(R)} d h_{k} d s_{k}
\end{aligned}
$$

$$
\begin{aligned}
& f\left(r_{k} / H_{0}\right)=\int_{0}^{+\infty} \frac{2 h_{k} e^{-h_{k}^{2}}}{\sqrt{2 \pi \sigma_{w_{k}}^{2}}} \exp \left[-\frac{\left(r_{k}+\sqrt{P_{r k}^{f c}} h_{k}\right)^{2}}{2 \sigma_{w_{k}}^{2}}\right] d h_{k}\left(1-\sum_{n=1}^{M} \frac{\tau_{k}^{n-1} \Gamma(R+n-1)}{\Gamma(n)\left(1+\tau_{k}\right)^{R+n-1}}\right) \\
& +\int_{0}^{+\infty} \int_{\tau_{k}}^{+\infty} \frac{2 h_{k} e^{-h_{k}^{2}}}{\sqrt{2 \pi \sigma_{w_{k}}^{2}}} \exp \left[-\frac{\left(r_{k}-\sqrt{P_{r k}^{f c}} h_{k} s_{k} / \tau_{k}\right)^{2}}{2 \sigma_{w_{k}}^{2}}\right] \frac{s_{k}^{M-1} \Gamma(M+R)}{\left(1+s_{k}\right)^{M+R} \Gamma(M) \Gamma(R)} d h_{k} d s_{k}
\end{aligned}
$$

\section{Fusion rules}

The received discrete signals by the FC from all the K sensors can be denoted by $\mathbf{r} \square\left[r_{1}, r_{2}, \cdots, r_{K}\right]^{T}$.The optimal test in Neyman-Pearson sense for the problem above can be formulated as $\quad \Lambda_{\text {opt }} \square \ln \left[\frac{p\left(\mathbf{r} \mid H_{1}\right)}{p\left(\mathbf{r} \mid H_{0}\right)}\right]_{H_{0} \text { is true }}^{H_{1} \text { is true }} \underset{\gamma}{\lessgtr} \quad$, where $\Lambda_{\text {opt }}=\left[\sum_{k=1}^{K} \ln f\left(r_{k} / H_{1}\right)-\ln f\left(r_{0} / H_{1}\right)\right]$ and $\quad \gamma \quad$ denote the log-likelihood ratio and the threshold chosen to assure a fixed system false-alarm rate, respectively.Considering that the optimum detector is mathematically 
intractable, we turn to the suboptimum EGC fusion rules [3]. The fusion rule of EGC is given by $\Lambda_{E G C}=\sum_{k=1}^{N} r_{k}$.

\section{Simulation Results}

We consider an eight-sensor system for the following three cases. In case I, the local sensors have the same performance index, i.e., $P_{l d k}=0.5$ and $P_{l f k}=0.01$ for all k’s, and the channels have the same average received SNRs.

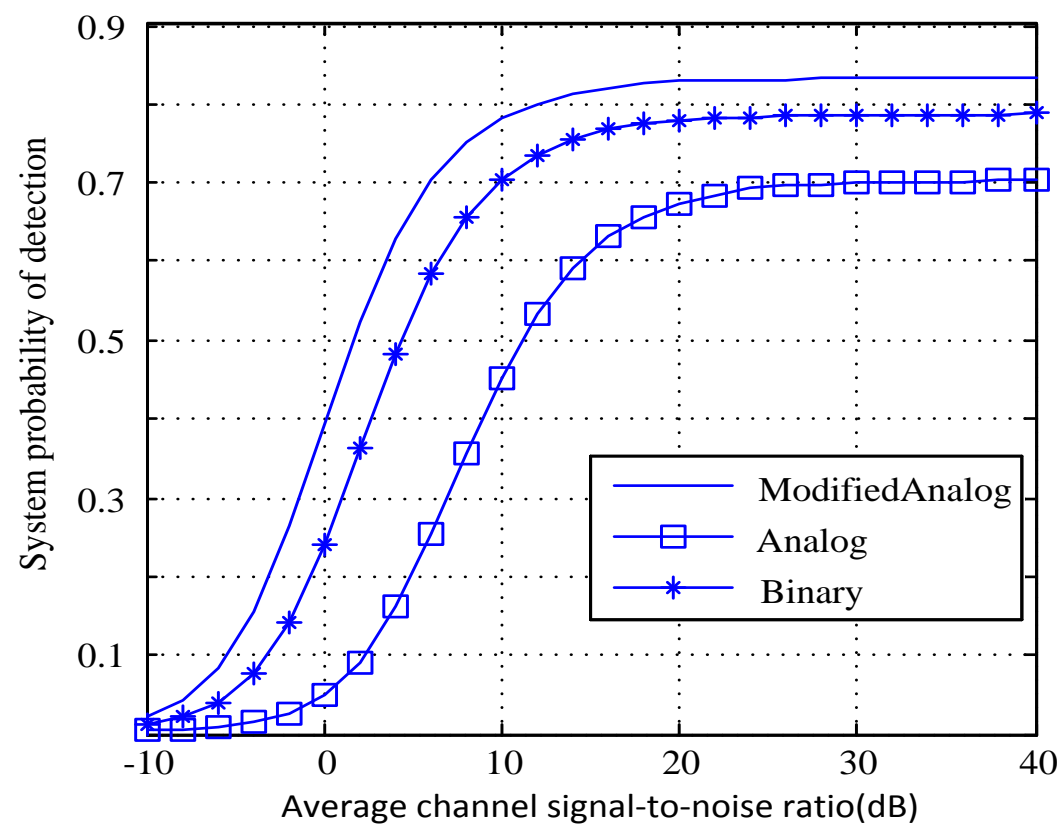

Fig.1 System probability of detection versus the average received SNR in case I

In case II, the local sensors have different detection performances, but the channels have the same average received SNR. Specially, $\left\{P_{l d k}\right\}=\{0.1,0.1,0.3,0.3,0.5,0.5,0.7,0.7\}$, and $P_{l f k}=0.01$ for all k's. In case III, the local sensors have the same performance index as case II, but the channels have disparate average received SNRs. Specially, the average received SNRs of the channels are distributed as 
$\left\{\mathrm{SNR}_{k}\right\}=\{S+2, S-2, S+4, S-4, S, S, S+3, S-3\} \mathrm{dB}$, respectively, where $S$ denotes the arithmetic mean of $\left\{\mathrm{SNR}_{k}\right\}$ in decibels.

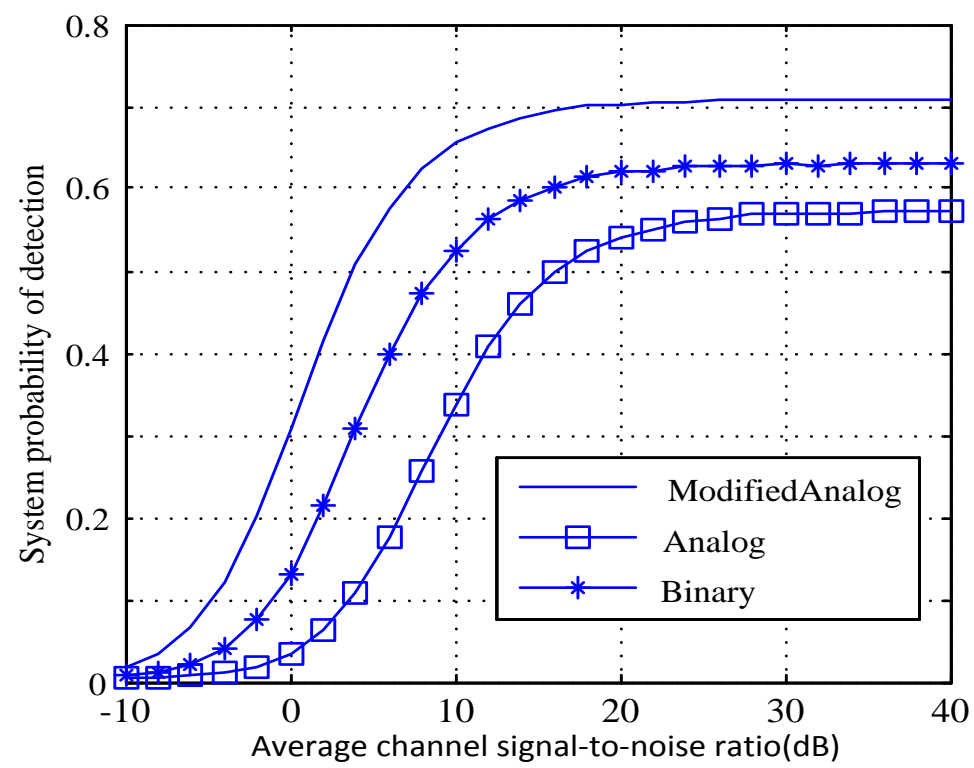

Fig.2 System probability of detection versus the average received SNR in case II.

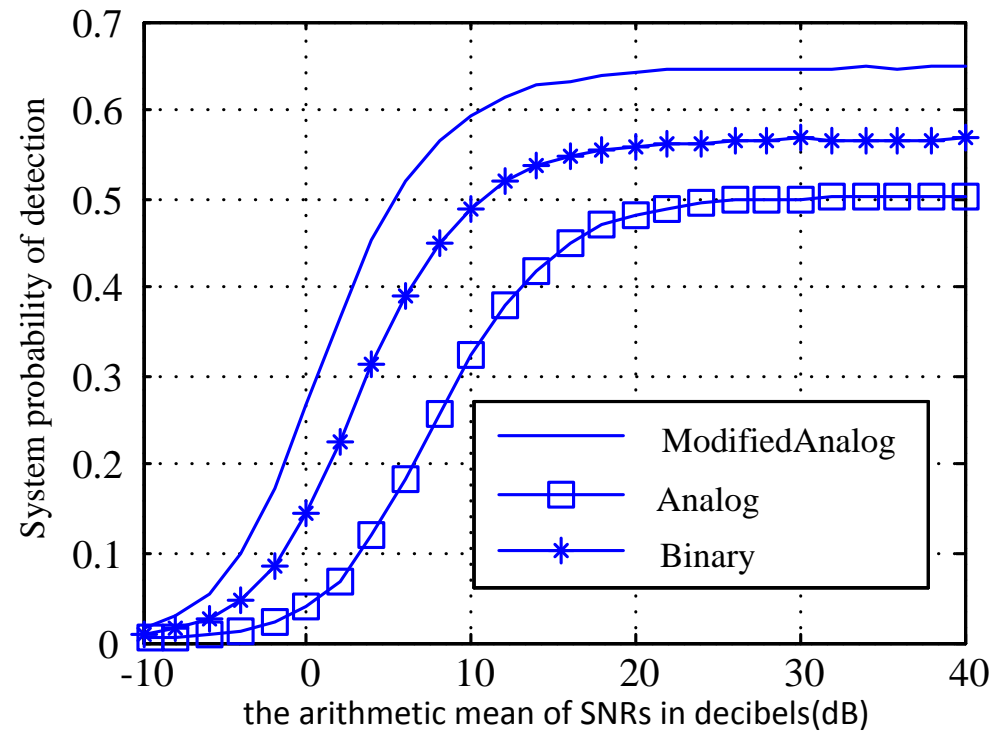

Fig. 3 System probability of detection versus the average received SNR in case III. 
It is still a good choice to adopt the modified analog transmission scheme when local sensors have different performance indexes, as described in case II. The increase in detection probability is as much as 0.8 relative to the binary scheme, or as much as 0.16 relative to the simple analog scheme, as shown in Fig.2. Under disparate received channel SNRs of case III, the proposed modified analog transmission scheme also brings significant performance gain, as much as 15 percent relative to the binary scheme, or as much as 33 percent relative to the simple analog scheme, as shown in Fig.3. From Fig.1-Fig.3, we can make a conclusion that the proposed modified analog scheme can improve the system detection performance significantly compared with not only the binary scheme but also the simple analog one.

An interesting phenomenon can be observed that, when the channel SNR is larger than $20 \mathrm{~dB}$, the detection performance will almost keep constant. In another word, the detection performance of the distributed detection system is not proportional to the channel signal-to-noise ratio.

\section{Conclusions}

A modified analog transmission scheme for distributed signal detection was proposed and evaluated in this paper. It combines the simple analog transmission and the binary digital transmission. Simulation results show that, without increasing extra transmission costs, the proposed transmission scheme can achieve significant performance gain not only over that of the widely adopted binary transmission scheme but also over that of the simple analog transmission scheme. The proposed scheme, however, is of high performance.

\section{Acknowledgment}

The China National Science Foundation under Grant Nos. 61102160 and 61179002 support this work. 


\section{References}

[1] J.Y Wu, C.W. Wu and T.Y. Wang, Channel-Aware Decision Fusion With Unknown Local Sensor Detection Probability. IEEE Trans. on Signal Process., vol.58(2010), p. 1457-1463.

[2] B. Chen, R.X. Jiang, et al., Channel aware decision fusion in wireless sensor networks. IEEE Trans. on Signal Process., vol.52(2004), p. 3454-3458.

[3] R. Niu, B. Chen, and P.K. Varshney, Fusion of decisions transmitted over Rayleigh fading channels in wireless sensor networks. IEEE Trans. on Signal Process., vol.54(2006),p. 1018-1027.

[4] K.C. Lai, Y.L. Yang and J.J. Jia, Fusion of Decisions Transmitted Over Flat Fading Channels Via Maximizing the Deflection Coefficient. IEEE Trans. Veh. Technol.,,vol.59(2010), p. 3634 - 3640.

[5] M. MARTAL O, and G. FERRARI, Decoding and Fusion in Distributed Detection Schemes with Unreliable Communications. IEEE Trans. Aerosp. Electron. Syst., 2012. 48(1): p. 16-26.

[6] S.K.Jayaweera, Large System Performance of Power-constrained Distributed Detection with Analog Local Processing, in 2005 ICWNCMC.(2005), p. 1083-1088.

[7] Wimalajeewa, T. and S.K. Jayaweera, Power efficient analog forwarding for correlated data fusion in wireless sensor networks, in 2007 IEEE 66th VTC.(2007), p. 367-371.

[8] Fadhil, A.M., H.M. AlSabbagh, and T.Y. Abdallah, Fusion Performance in Wireless DS-CDMA Sensor Networks with Analog Relay Local Processing Scheme. network, 2012. 5(3): p. 141-150.

[9] M. Gastpar, B. Rimoldi, and M. Vetterli, To Code, or Not to Code: Lossy Source-Channel Communication Revisited. IEEE TRANS. ON INFORMATION THEORY, vol.49(2003), p. 1147-1158. 